\title{
GASTRECTOMY FOR PEPTIC ULCER
}

\section{Selection, Management and Care of Patients}

\author{
By M. Gamal Eldin, M.B., F.R.C.S., Ed., F.R.C.S., EnG., and \\ JOHN W. WARRICK, M.R.C.S., L.R.C.P., D.A.
}

\section{St. Alfege's Hospital, Greenwich}

The majority of gastrectomies is carried out for chronic peptic ulceration, whether gastric or duodenal, which fails to respond to, or recurs more than twice after, a strict ulcer regime has been instituted. A patient may be regarded as a candidate for operation if, after one or two years' treatment he still suffers from the ulcer. During that time 2 to 3 diet regimes should have been tried with intervals of 3 to 6 months as necessary. Repeated small haematemeses or melaena, or more than two severe haemorrhages are other indications for operation. Penetration into the pancreas is usually accompanied by such constant severe pain often referred to the back that the unfortunate patient begs for an early operation.

For chronic gastric ulcers all agree that gastrectomy and not gastrojejunostomy is the operation of choice. These ulcers heal less readily than those in the duodenum and about ro to 20 per cent. become malignant.

In chronic duodenal ulceration, however, gastrojejunostomy has been adopted by some surgeons as the better operation because duodenal ulcers tend to heal more rapidly than gastric ulcers, they do not become malignant and the operative mortality is low. The tendency now is to perform gastrectomy for duodenal ulcers as results are more satisfactory and permanent, the ulcer bearing area being removed; the acid stimulating area of the stomach is diminished and the tendency to anastamotic ulceration is minimized. Furthermore the mortality of the operation is little more than that of gastrojejunostomy. The indications for gastrojejunostomy nowadays should be pyloric obstruction or a very scarred and adherent duodenal ulcer in a patient who is a poor operative risk.

The preliminary investigations are usually carried out in the Medical Out-Patients Department. These include general and specific examination to assess the patient's fitness for a major operation and to determine the nature, position, etc., of the ulcer.

In the general physical examination particular attention is paid to the cardio-vascular and respiratory systems. Hypertension, unless it is of the malignant type or accompanied by severe renal dysfunction, does not preclude operation. Red cell count and haemoglobin percentage are estimated and any degree of anaemia corrected. The blood group is determined in case serious bleeding necessitates transfusion later. Respiratory complications are the commonest cause of trouble after upper abdominal operations. Any serious pre-existing disease such as severe bronchitis or bronchiectasis is a contra-indication to gastrectomy. If surgery is essential it may be necessary to do the less formidable operation of gastrojejunostomy. Minor chest troubles such as mild bronchitis or a recent history of acute upper respiratory tract infection should be treated by aerosol penicillin inhalations.

The teeth should be in a healthy condition. Wholesale extraction is not a routine procedure and only loose or obviously septic teeth should be removed some days before admission to hospital.

The usual investigations on the stomach are carried out. A positive barium meal is of great value as it verifies the existence of an ulcer, or any other lesion, and shows its size, shape and position. Screening is more imimportant than filming as it not only discloses an ulcer which occasionally escapes a film, but also demonstrates the gastric behaviour as regards mobility and peristalsis. A lateral or oblique view is necessary to show a posterior 
or anterior wall ulcer. Negative barium meal is not definite proof of the absence of an uloer ; we have met with cases which on laparotomy carried out on purely clinical grounds showed posterior wall ulcers that failed to show radiologically. On the other hand deformity of the duodenal cap may mean a completely healed ulcer or adhesions around a healthy duodenum, i.e., from the gall bladder.

Fractional test meal (alcohol test preferred) should be carried out. The most valuable information is obtained from the acid curves. The degree of acidity should be a rough guide as to the correct operative procedure to be adopted and the extent of the gastric resection.

The value of gastroscopy before gastrectomy is debatable but in the average case where the clinical picture is typical and the X-ray findings confirmative gastroscopy is not essential. It is indicated when the clinical features are suggestive but repeated radiological investigations are negative. This is especially the case in small non-curvature ulcers (anterior or posterior wall ulcers).

The general behaviour of the patient should be such as to ensure co-operation after operation and it is always wise to explain to him beforehand the procedures such as passing a Ryle's tube that may be necessary when he is in hospital.

\section{Immediate pre-operative care}

It should be stressed that the less the patient is prepared and disturbed before the operation the smoother will be the postoperative period. The patient is admitted two days before the operation, having already had all the investigations considered necessary in his particular case. $\mathrm{He}$ is thus spared the inconvenience of further investigations in the immediate pre-operative period and his morale is not disturbed. $\mathrm{He}$ is allowed out of bed and encouraged to move about the ward; he is given a full diet for the first day but a lighter diet on the eve of the operation. He begins a course of breathing exercises soon after admission, which is continued after the operation. Soneryl two capsules $\left(4 \frac{1}{2} \mathrm{gr}\right.$.) is given at night time if required. On the morning of the operation he is given a cup of tea. Stomach lavage should not be carried out as a routine as it disturbs the patient unduly and serves no purpose other than to remove the antiseptic acid of the stomach. Likewise blood or plasma transfusion before or during operation is not recommended and is only indicated if there is severe haemorrhage during or after the operation.

\section{The Operation}

(a) Anaesthetic technique. In view of the duration of the operation it is considered that the patient should be unconscious throughout. Heavy premedication is not indicated since in all cases anaesthesia is induced with i.v. Pentothal and large doses of respiratory depressants are bound to do more harm than good. Omnopon gr. I/6 with Scopolamine gr. $1 / 300 \quad 1 \frac{1}{2}$ hours pre-operatively is quite satisfactory and there is no indication for a larger dose.

There are three periods during the operation when extreme relaxation is essential in order that the surgeon can work under the best possible conditions. These are during the preliminary intra-abdominal manoeuvres ; when the duodenal stump is being dealt with ; and during the suture of the peritoneum when the wound is being closed. During the anastomosis moderate relaxation only is necessary. At all times respiration must be quiet and unaccompanied by increased diaphragmatic activity. There are two techniques above all others which are particularly indicated since they combine the above criteria with minimal after effects.

i. Regional anaesthesia with light general anaesthesia.

ii. Curare with light general anaesthesia.

In either method after a Pentothal induction anaesthesia is maintained in a light plane with nitrous oxide. When regional anaesthesia is to be used the patient is turned on to his side and the thoracic nerves 6 to 12 are blocked at the angle of the ribs using 1 : 1000 Amethocaine-Adrenalin solution. Small additional amounts of Pentothal may be required during the course of the operation; it is better to do this or to add small amounts of Trilene or Cyclopropane than to lower the oxygen percentage should the plane of anaesthesia become too light. When curare is to be used anaesthesia is established as above and a small test dose of Tubarine mgm. 5 is given intravenously. Just before the operation begins 
the main dose of Tubarine mgm. $15-20$ is injected. It is essential to have a gas-tight fit between the face and the anaesthetic mask when curare is used; if this is difficult to obtain a cuffed endotracheal should now be passed. It is important that an adequate tidal exchange be maintained and aided respiration by manual compression of the rebreathing bag is a wise procedure and is essential if respiration is depressed. Further small injections of Tubarine mgm. 5-6 i.v. may be necessary before the duodenal stump is dealt with and before the peritoneum is sutured. Should there be any degree of respiratory depression at the end of the operation Prostigmine mgm. 5 with Atropine gr. 1/50 is injected i.v.

Observation of the blood pressures and pulse rate shows that with regional analgesia there is little alteration in these figures both initially and during the operation. When curare is used the systolic pressure tends to rise without corresponding change in the diastolic figure. When Cyclopropane is the inhalation anaesthetic there are often variations in the pulse rate; the reason for this is uncertain and does not appear to have any definite relation to the depth of anaesthesia.

The degree of muscular relaxation can readily be determined by the ease with which the patient's expiratory efforts can be overcome by manual compression of the rebreathing bag.

(b) Operative technique. The incision is a matter of choice, but the median incision-is good as it is quicker to make and to close and allows better retraction and equal exposure of both sides of the abdomen. The usual, but unfair, objection to it is the alleged more frequent sequela of incisional hernia. The main cause of incisional hernia is inadequate closure of the peritoneal layer leaving gaps or perhaps tears which invite the intestine or omentum to glide through and work its way out until it lies under the skin. This could occur in any incision in which there were peritoneal gaps. The linea alba is made of scar tissue. The incision heals by scar tissue which should restore the normal anatomy of the area. Thus it is more natural to perform the median than the paramedian incision. In our experience results of median incision are excellent.
The operation should be bloodless however difficult the case may be if the omenta with their unsupported vessels are handled gently and are cut between two Spencer Wells. The greater omentum should be dealt with first, starting preferably nearest the fundus as it is much easier to get into the lesser sac in this region. Care should be taken lest the mesocolon should be adherent to the omentum and be cut with it. Not uncommonly another folded layer of peritoneum is found in the lesser sac between the posterior wall of the stomach and the pancreas and is mistaken for adhesions. This may cause confusion but it is bloodless and should be easily snipped without bleeding.

The amount of stomach removed varies but should include the lesion present whether gastric or duodenal. In the average case the line of section in the stomach is indicated on the greater curvature by the region where the right and left gastroepiploic vessels end by turning up on the surface of the stomach without obvious direct anastomosis.

Utmost care should be exercised in closing the duodenal stump as many post-operative deaths are due to its leak or rupture. A very secure procedure is to close it with a running through-and-through suture under the occluding duodenal clamp-which is then removedand then followed by Lembert's invaginating suture. Should the area be very fibrosed the duodenum could be cut across without clamps, the mucous membrane identified and closed with a continuous suture and this finally covered over by the tissues around. A penetrating gastric ulcer should be separated boldly from the pancreas without fear of bleeding. The resulting perforated area on the surface of the stomach should then be closed momentarily by a deep mattress stitch.

One of the difficulties in dealing with high gastric ulcers is the application of occluding clamps above the ulcer area. The clamp can be dispensed with by cutting across the stomach with the cautery thus preventing bleeding, and sucking out the stomach contents, thus avoiding soiling, after fixing the posterior seromuscular layer between the posterior gastric surface and the jejunum. The jejunum likewise should be opened with the diathermy. We do not use rubber on clamps 
as it makes them cumbersome. Contrary to general belief uncovered clamps if properly used do not harm the tissues more than the covered ones.

A modified anterior Polya operation (Hoffmeister's technique) gives good results. Catgut is used throughout the operation. The incision is closed in two layers, tension sutures being fixed after closing the peritoneal layer. Should the peritoneum tear, the peritoneum and linea alba should be closed in one layer with interrupted catgut. No peritoneal gaps should be overlooked. The wound is covered with gauze fixed with elastoplast. The elimination of bandages and binders in $\mathrm{ab}$ dominal surgery has done much to lower the incidence of post-operative chest and thrombosis complications.

\section{Post-operative Care}

(a) Immediate. When curare has been used morphia should be given as soon as the patient returns to the ward. It is repeated the same evening. Morphia, if indicated, should never be withheld during the first 48 hours; it relieves pain and anxiety and promotes local and general rest.

As mentioned above breathing exercises are continued until the patient is out of bed on or about the I2th day. Should there be any unexplained rise of temperature or pulse rate the chest is X-rayed in order to determine whether there is any degree of atelectasis.

During the first 24 hours nothing is given by mouth, which, however, should receive due hygienic care. Fluid should be provided by rectal water given slowly. and in the same rate and manner as an intravenous drip. It is simple to administer and is always tolerated if given slowly. Intravenous saline is not recommended as the patient has not lost any chlorides ; the only indication for intravenous saline should be excessive vomiting. Stomach washouts are not allowed unless dilatation of the stomach or bleeding is suspected as indicated by repeated vomiting. We have rarely had to pass a Ryle's tube after gastrectomy much to the comfort of the patients.

As a routine we give patients gms. I of Sulphamezathine 4 hourly for 2-3 days, the first dose starting with the first feed 24 hours after operation. This acts locally by passing over the suture line, and generally after absorption ; thus it is a good prophylactic against local and general infections, including respiratory troubles.

Diet in the second 24 hours is made up of water I ounce hourly for 6 hours; whey or water 2 ounces hourly for 6 hours, and then 3 ounces hourly for 12 hours. On the third day citrated milk and water in 4 ounce feeds alternatively with whey hourly for 24 hours. Next milk and egg and chicken broth are allowed, and later boiled eggs, steamed fish, bread and butter, custard and jelly are added to the diet.

Stitches are taken out on the 8th day and the patient leaves his bed on the 12th day, but he is allowed and encouraged full freedom of movement all the time. $\mathrm{He}$ is discharged on the I $4^{\text {th }}$ day.

Instructions should be given to the patient that for 4-6 months he should not eat big meals or he may get mechanical vomiting because of the small size of his stomach as a result of the operation. The stomach will, however, gradually resume its normal size. Some of those cases neglecting the above instructions have been corrected successfully by eating small frequent meals.

After a large resection of the stomach the patient may complain of indigestion or gastric pain. This may be due to achlorhydria following the operation as shown by fractional test meal. It responds well to the intake of IO-I 5 minims of Acid Hydrochloric Dilutum (B.P.) to each meal. 